\title{
Structuring user interfaces with a meta-model of mental models
}

\section{Christian Sifaqui*}

Technische Universität Darmstadt, Fachbereich Informatik, Fachgebiet Graphisch-Interaktive Systeme, Rundeturmstraße 6, D-64283 Darmstadt, Germany

\begin{abstract}
This article presents a meta-model of user interfaces, based on the idea of mental models. This meta-model provides its utility in single-user environments as well as those for multiusers. A prototype is presented that implements the meta-model and two trial areas are chosen for discussing its operation. The most innovative aspect is unifying different use schemes in one interface, like temporary, spatial, networked or structured data organizations. (C) 1999 Elsevier Science Ltd. All rights reserved.
\end{abstract}

Keywords: User interfaces; Mental models

\section{Introduction}

Taste and behaviour of each individual differs. This simple observation is presented in every action of each individual. Each individual, therefore, interacts in a different way, in congruency to his model of the world and to his previous experiences (see Fig. 1(a)).

When incorporating a new element (the computer) in the interaction, the model of the world of the individual expands and its model generates new models of thought as well (see Fig. 1(b)). Following Llinás [1] the human being owns information and a processing capacity in an "a priori" state. During the interaction with the environment this capacity achieves an "a posteriori" state that depends on the interaction and the archived information.

What has been previously described is intimately related with the Activity Theory and the concept of mental models that are detailed next.

\subsection{Activity Theory}

The human being when interacting with a tool varies his or her mental model and model of the external world. This idea forms the centre of the Activity Theory [2-5].

\footnotetext{
* Tel.: + 49-6151-155-609; fax: + 49-6151-155-196.

E-mail address: sifaqui@igd.fhg.de (C. Sifaqui)
}

The Activity Theory originated in the former Soviet Union as part of the cultural-historical school of psychology founded by Vygotski, Leontjev and Lurija. The theory is a philosophical framework for studying different forms of human praxis as developmental processes, with both the individual and social level interlinked. The fundamental unit of analysis is the human activity which has three basic characteristics: firstly, it is directed towards a material or ideal object which distinguishes one activity from another; secondly, it is mediated by artifacts; and thirdly, it is social within a culture [6].

Human activity can be described as a hierarchy: activities realised through chains of actions, which are carried out through operations. Human activity is always directed towards a material or ideal object satisfying a need and the subject's reflection of, and expectation to, this object characterises the motive of the activity.

Following this idea we can have physical tools that manipulate and vary the external world, and psychological tools that manipulate and vary thinking. In this way, the computer, unlike many other artifacts, guarantees both services.

\subsection{Mental models}

As a result of the interaction with the world, the human being builds internal ideas of it. These representations in the mind are called mental models. 


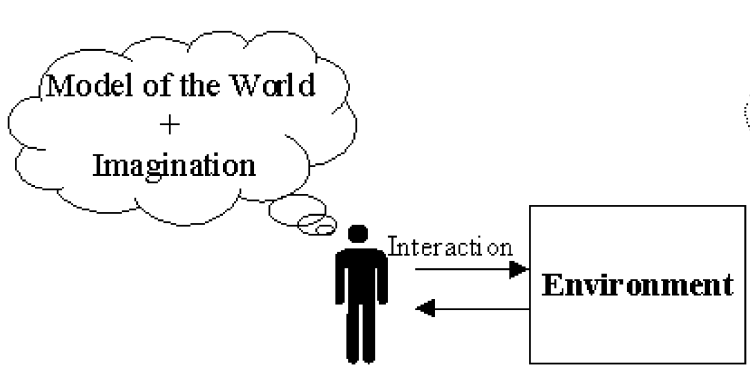

(a)

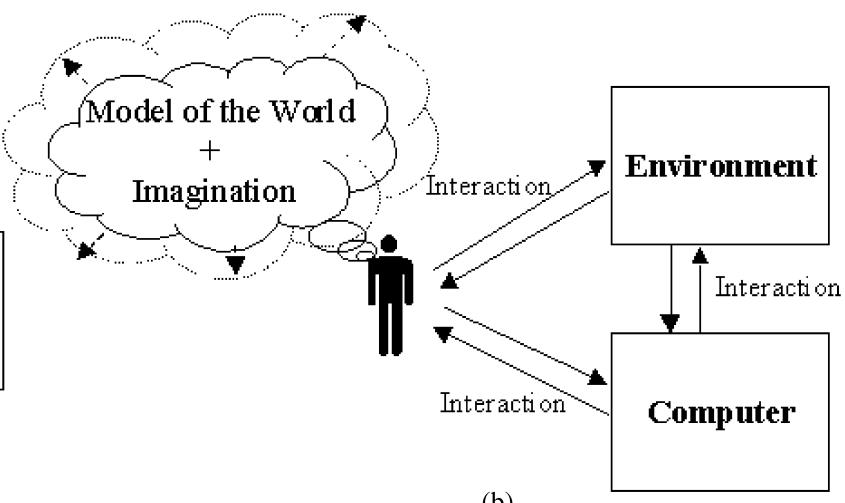

(b)

Fig. 1. Human behaviour.

The term "mental model" has been used for completely different models or ideas, which led to a discussion during the 1980s.

Within the computer area it is assumed that the users make use of "mental models" in order to understand and to be able to predict the behaviour of a system. The success in using a system depends on how well the mental model represents the system. Carrol and Olson [7] describe the confusion about the use of the term "mental model" and conclude that the most frequent use is:

a representation (in the mind) of a physical system or software being run on a computer, with some plausible cascade of causal associations connecting the input to the output.

In accordance with Staggers and Norcio [8], many authors consider the mental models as organized structures, consisting of objects and their combinations. On the other hand, Redish [9] suggests that mental models consist of propositions, pictures, procedure rules and statements and indications of how and when they may be used. Rauterberg [10] proposes that mental models should contain three different kinds of knowledge: the logic structure of the work, the sequential structure of the targets and the temporal structure of all operations.

In this paper, a mental model is the manner the user carries out his or her activities (with or without a computer) and how he or she organizes the information in his or her memory. In some manner this corresponds with the definition given by Lokuge et al. [11]:

the cognitive layout that a person uses to organize information in his or her memory.

Following these assumptions, a new way of structuring and designing user interfaces is suggested that dynamically accommodates mental models to represent the structured information.

\section{Presentation of a meta-model}

In accordance with the theoretical foundation of the introduction we could outline a meta-model (see Fig. 2) that is based on the results of the work of Barreau and Nardi $[12,13]$ as well as on the results of the field of research of the Neuroscience [14] that accomplish the creation of computational mental models basing on the Activity Theory.

\subsection{Architecture of the meta-model}

Fig. 2 represents in a simple form what the meta-model looks like.

This configuration makes it possible that the user, by means of his or her model of thought, models his or her "Infoverse".

The meta-model consists of three levels:

- level A deals with the representation of the information;

- level B takes care for the modeling of the mental models;

- level $\mathrm{C}$ is the long-term memory of the information.

This model stores the semantic information into three different databases. So we have a database with ephemeral information, which only serves to deal with the actual representation to the user. It is ephemeral, because the user can change the representation according to his or her needs.

Next, we have a database with working information, this information models the actual computational mental model, when the user changes his or her computational mental model, some information goes to the archived information database.

Finally, we have the archived information, which stores the rules, procedures that do not vary during the use. 


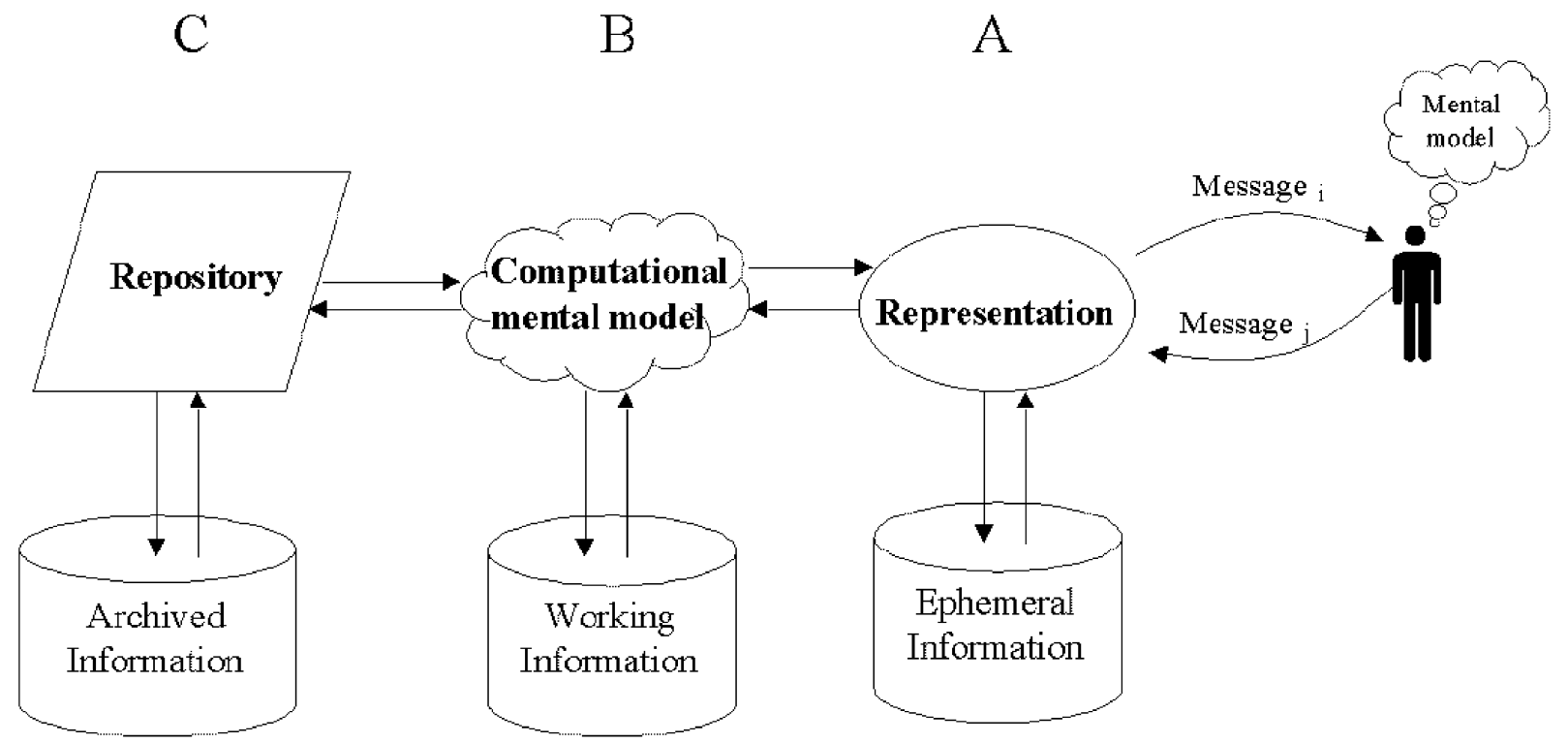

Fig. 2. Meta-model.

\subsection{Individual or shared use of the meta-model}

This meta-model allows an individualized interaction with every level, where each level is adapted to a single user (see Fig. 3); or a shared interaction that can be carried out in three different ways (see Fig. 4):

(a) the users refer to the same mental model and the same permanent storage area, their representation method differs;

(b) the users refer to the same permanent storage area, their representation method and their mental models differ;

(c) the three levels are individual, but there is a sharing of information from the permanent storage area of each user.

\subsection{A proposed meta-organisation of the meta-model}

As a general structure to deal with the three levels of the meta-model the concept of "knowledge plane" is proposed where every knowledge plane represents a structure similar to a "Mind Map" [15]. Every plane consists of "ideas" and combinations or links between the "ideas". In addition an "idea" can be placed in different planes and also different planes can be combined.

The key is that every plane contains ideas that have something in common.

The ideas can be seen as simple files and the associations between these as simple references, and thus one can consider the plane as a directory. The other possibility is that an idea can be seen as hypertext nodes and the

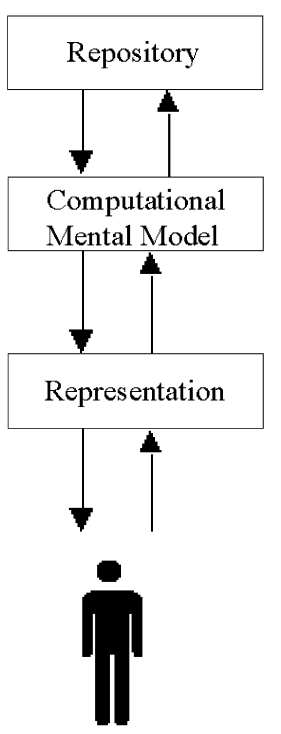

Fig. 3. Individual use of the model.

associations as links and thus the plane is a sort of a "web". Also the ideas can procedure rules and the associations between these can have a temporal gap, giving as a result that the plane can be viewed as a "workflow". Furthermore, there is the possibility that the combinations between the ideas have procedure rules, propositions or statements leading to a completely different structure.

In this way, the "knowledge planes" are more general than an organization of files into tree structures (like 


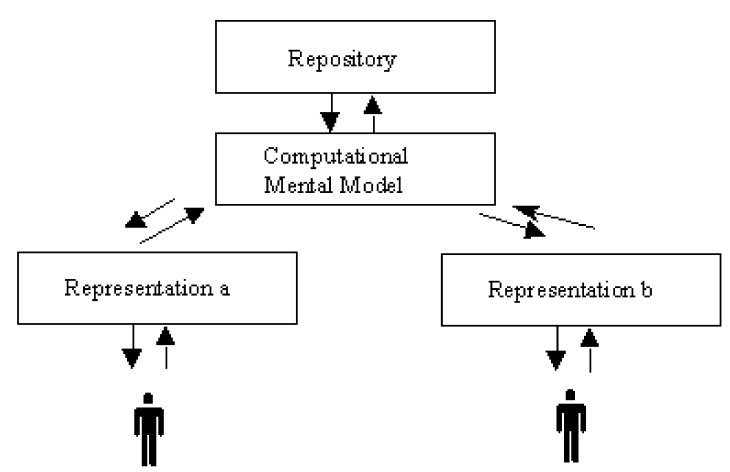

(a)

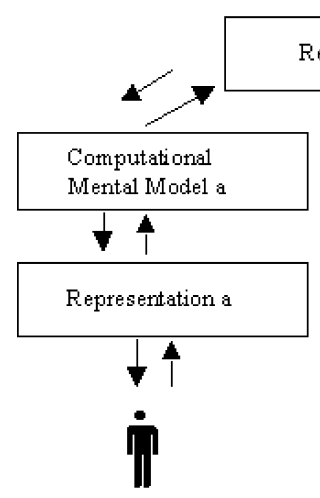

(b)

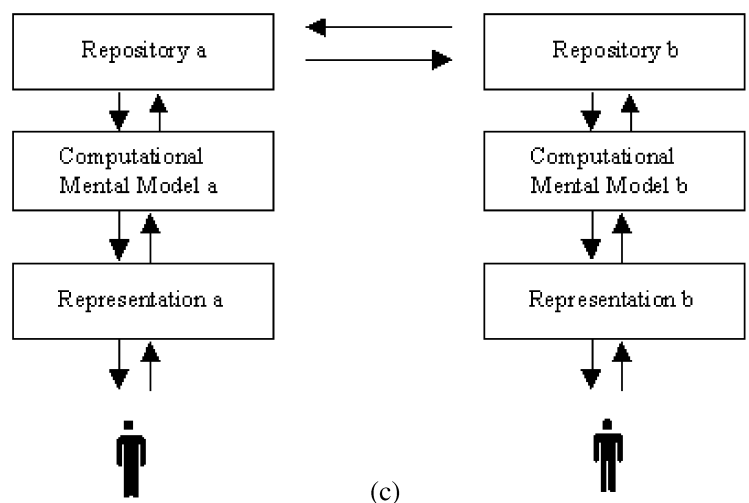

Fig. 4. Shared use of the model.

MS-DOS), dag (direct acycled graph, like UNIX) or temporal organization based on streams (like the proposal of Lifestreams [16]). Perhaps "The Brain" methaphor [17] would be the nearest representation, but it offers no possibility to group collections of similar ideas in a common structure and it does not support more than a simple combination of "thoughts".

A draft visualization of this representation is given in Fig. 5, where the ideas are represented by the nodes on each plane.

\section{Design and implementation of a system based on the meta-model}

Since 1997 a prototype that implements the metamodel has been under development. BACán is implemented in Java3D and uses XML [18] for data modelling.

As it is based on Java, the system offers the advantage of being independent of the platform. In addition, by its conception of an open system, the use of applications specific to the platform is stimulated which the user is accustomed to.

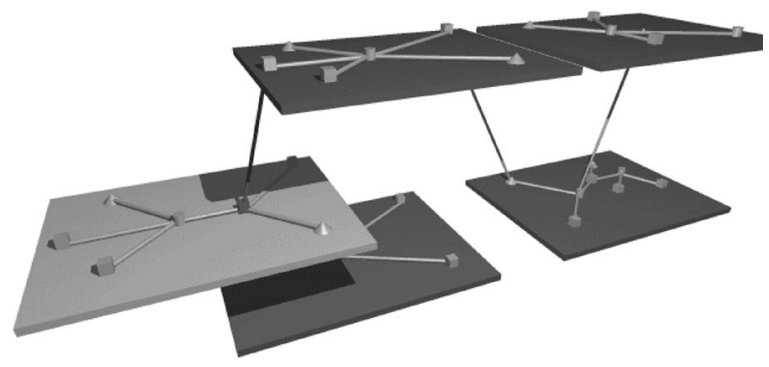

Fig. 5. Knowledge planes.

In the following, the architectonic details of the implementation of each level of the meta-model in the prototype are described.

\subsection{Implementation of level $C$}

Level $\mathrm{C}$ has two basic functions:

- being source of the basic information of the system;

- coding and encapsulating pre-existent information. 
For this reason, the "archived information" database is made up of pre-existing information that has been codified and encapsulated by means of XML.

The action rules and a XML parser are in the "repository" that in turn maintains and generates the link meta-data structures which correspond to the "knowledge planes".

Let us suppose the previous existence of a $\mathrm{LAT}_{\mathrm{E}} \mathrm{X}$ file with its bibliography file, then the encapsulation modules codify these two files so that they are used in a knowledge plane later that models the process of editing, compiling, visualizing, and printing a $\mathrm{LAT}_{\mathrm{E}} \mathrm{X}$ file (this additional complexity of $\operatorname{LAT}_{\mathrm{E}} \mathrm{X}$ makes it useful as example).

\subsection{Implementation of level $B$}

The computational mental model stores predefined rules (as the process of manipulating a $\mathrm{LAT}_{\mathrm{E}} \mathrm{X}$ file indicated in the previous section) or new rules defined by the user to model the behaviour of some "knowledge plane".

Furthermore, this computational mental model maintains an information basis about the user's preferences, that is, a user model $[19,20]$ to carry out the presentation of the knowledge planes and to invoke the external applications related to the platform of execution.

The working information database consists of a set of meta-data files, and the rules, procedures or statements, as well as the storage of the invariant user preferences.

Due to the dynamic nature of the mental models, this level allows being adjusted by the user or automatically being adapted by the system based on his or her interactions with the system.

\subsection{Implementation of level $A$}

The representation basically consists of a 3D navigation in the knowledge planes. According to the type of plane visualization can be provided. The visualization consists of a simple implementation of the table proposed in chapter 15 of [21]. Because there are many visualization procedures, each user can adapt the visualization to his interest or his advantages (supporting the fact that every user has different capabilities to perceive his environment [22]). To support the preceding idea, the prototype provides a "layer" mechanism, which the user selects. This "layer" can be seen as a particular implementation of the Magic Lens [23-26] style.

According to the user model, the ideas can be processed by the applications preferred by the user, an example would be to edit a $\mathrm{LAT}_{\mathrm{E}} \mathrm{X}$ file with $v i$ if one is working on the UNIX platform and prefers $v i$.

The ephemeral information database stores only temporal files necessary for the visualization purposes and provides the necessary modules to capture the user behaviour.

\section{Examples}

Two examples for the usage of BACán are presented. These examples have been chosen to show the usage of BACán in situations where the mental model of each user was intensely present.

\subsection{Use of the system as workflow}

Nowadays the handling of the information can be carried out in a manual or automatic form. The processing of this information can be defined in work steps which forms the business process. These business processes can be modelled in an automatic form by workflows.

When using a business process, each person involved has a mental model of how the activity works according to his or her view (analogous to a user view in database modeling [27]).

The interaction with a workflow can be described as situated planning [6] which has its roots in the Activity Theory.

\subsubsection{A little background}

Workflow systems have been addressed to by several authors as ways of routing information objects among users, and to specify automatic actions to be typically taken in that routing according to certain process models [28-30]. The workflow systems have been subject to much controversy and criticism for their rigid representation of work in process models [31,32]. According to the Activity Theory, the human activity is realised through chains of actions which are carried out through operations. These actions are controlled by the subject's conscious goals, the manner the human activity realises objective results. This was already suggested in a more detailed form by Norman [33] (p. 42).

Since the basic idea of a workflow is "any task performed in series or in parallel by two or more members of a workgroup to reach a common goal", we have the basis that a workflow must be done by a group of persons, where each person has his or her own personal mental model of the global activities. Thus workflow systems are a good subject of evaluation of the prototype.

\subsubsection{Use}

As an example, the process of writing a paper is presented. As Press [34] states, by writing a paper several stages are passed: brainstorming, writing and reviewing. These stages can be modelled as Fig. 6 shows: we have three linked knowledge planes. The first supports the brainstorming process and it serves to annotate rough ideas and their possible reference literature in a manner as stated in Card and Moran [35].

With these rough ideas and the reference literature, two files are generated, a $\operatorname{LAT}_{\mathrm{E}} \mathrm{X}$ and a bibtex file. These 


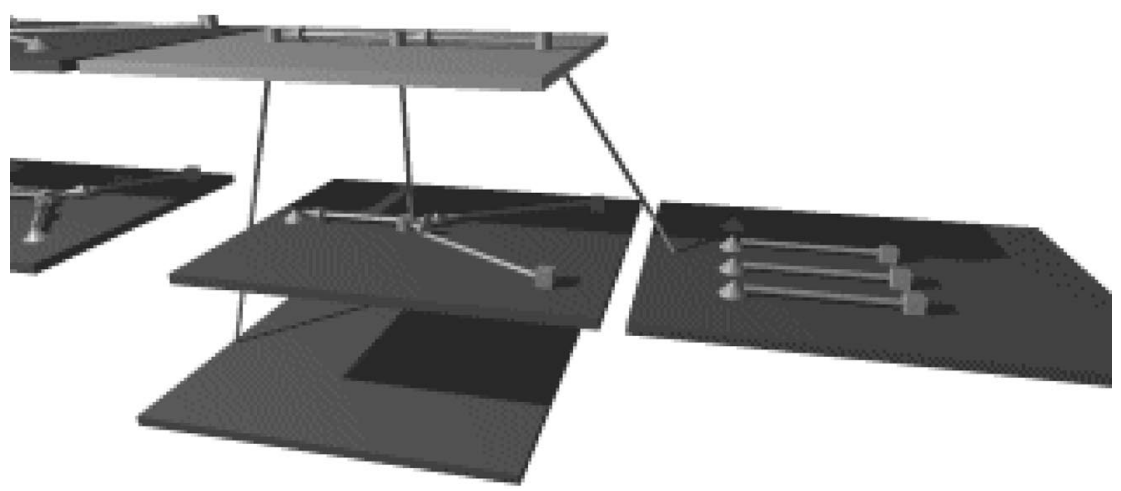

Fig. 6. A mental model of a workflow.

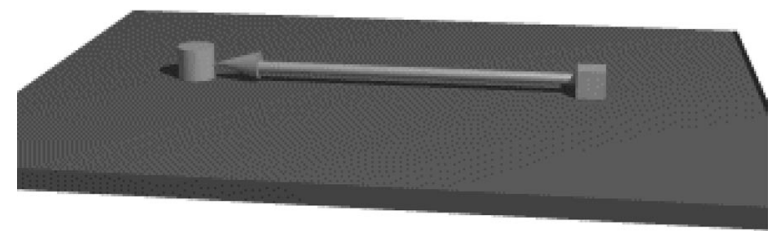

Fig. 7. Another mental model from the workflow.

two files are contained on a knowledge plane that models the processing of writing any $\operatorname{LAT}_{\mathrm{E}} \mathrm{X}$ file.

The next knowledge plane is the reviewing of a paper. This is done for another person who makes observations and comments and then gives them back to the author (by giving the knowledge plane).

Fig. 7 shows the knowledge plane for another person who makes the reviewing process.

\subsection{Use of the system as a program visualizer}

Diagrams, e.g. flowcharts, trees for hierarchical structures, or graphs for finite state machines, are often needed as part of visual systems and advanced user interfaces and are frequently application-specific.

\subsubsection{A little background}

An easily comprehensible representation of complex structures is desirable in every application and especially in visual systems. Using some kind of diagram as a graphical representation of the complex structures is often the best choice. Wellknown examples are NassiShneiderman charts [36], flowcharts, or finite state machines. We use the Nassi-Shneiderman diagram representation, which is not incorporated in prominent software products, but which successfully serves as a visualization aid in teaching novice programmers. NassiShneiderman has been chosen, because they have proved to be useful for program presentation and also scale for large problems. They provide a bird-eye view when "zooming out" of the diagram [37]. Contents of single statements might get lost, but the overall structure remains and even becomes more apparent. Furthermore, because of their block structure, these charts provide easy means of abstraction: complex blocks can be represented as simple statements. The complex structure is then elaborated in another chart.

\subsubsection{Use}

According to the Activity Theory, the act of programming would be directed by the goals of having an operative program, and the result of the execution of the program must correspond with this goal.

As it has been said in Section 3.2, a plane could be a logical grouping of the necessary files to make a program, as well as the representation of the sequence of steps to carry out a compilation.

By this way, the visualization of the program through a graphic representation can represent the way in which the goals are carried out, that is, it allows to represent a mental model of the program and of the process of programming.

As Rassmussen [38] states, a person has more than one mental model for an activity, a visualization that sustains this statement can be proposed. Fig. 8 shows a zoom on a node of a plane, that is, a program that is visualized as Nassi-Shneidermann chart.

\section{Conclusions}

In this article, it is shown how a new user interface system can be designed to meet the real needs of an individual or a team. The development is based on the mental model concept, which has not been totally defined until now. But even so, it serves to sustain a development base to model the activities that are carried out within the area of personal work, tele-work and its coordination of activities. 


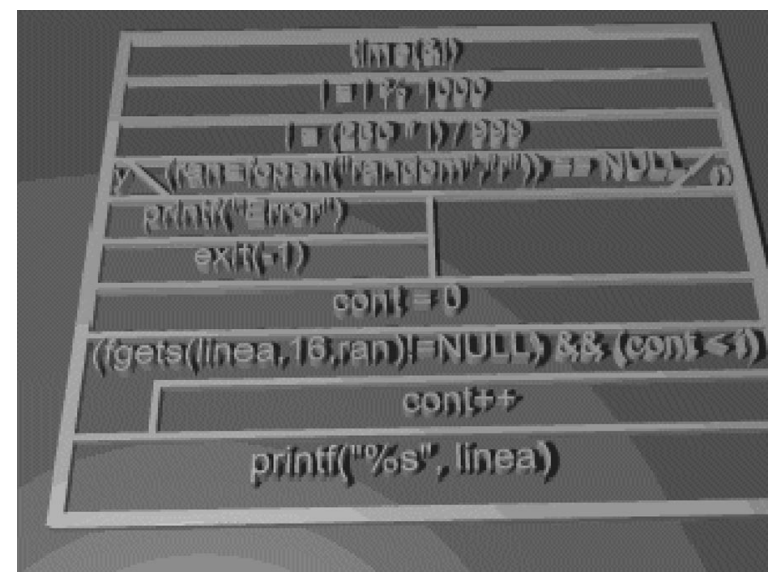

Fig. 8. Visualizing a program.

\section{References}

[1] Strejilevich S. Consciousness and the brain: interview with R. Llinás, Brain \& Mind. Electronic Magazine on Neuroscience, 1998;2(6). WWW address http://www.epub.org.br/ $\mathrm{cm} / \mathrm{n}$ 06/opiniao/llinas_i.html.

[2] Bødker S. Through the interface: a human activity approach to user interface design. NJ: LEA: Hillsdale, 1991.

[3] Nardi B, editor. Context and consciousness, activity theory and human-computer interaction. Cambridge: MA: MIT Press, 1995.

[4] Vygotsky L. Mind in society: the development of higher psychological processes. Cambridge: MA: Harvard University Press, 1978.

[5] Vygotsky L, Kozulin A. Thought and language, rev'd ed. Cambridge: MA: MIT Press, 1986.

[6] Bardram J. Plans as situated action: an activity theory approach to workflow systems. Proceedings of ECSCW '97 Conference, Lancaster, UK, September 1997.

[7] Carrol J, Olson J. Mental models in human-computer interaction. In: Helander M, editor. Handbook of human-computer interface. Amsterdam: North-Holland, 1988. p. $45-65$.

[8] Staggers N, Norcio A. Mental models: concepts for human-computer interaction research. International Journal of Man-Machine Studies 1993;38(4):587-605.

[9] Redish E. The implications of cognitive studies for teaching physics. The American Journal of Physics 1994;62(6):796-803.

[10] Rauterberg M. From novice to expert decision behaviour: a qualitative modelling approach with petri nets. In: Anzai Y, Ogawa K, Mori H, editors. Simbiosis of human and artifact. Amsterdam: Elsevier, 1995. p. 449-54.

[11] Lokuge I, Gilbert S, Richards W. Structuring information with mental models: a tour of Boston. Proceedings of CHI'96, Vancouver, BC, Canada, April 1996, p. 413-19.

[12] Barreau D, Nardi B. Finding and reminding: file organization from the desktop. SIGCHI Bulletin, 1995;27(3):39-43.

[13] Nardi B, Barreau D. Finding and reminding revisited: appropiate metaphors for file organization at the desktop. SIGCHI Bulletin 1997:29(1):76-8.
[14] Cardoso S. Remembering and forgetting, Brain \& Mind. Electronic Magazine on Neuroscience, 1997:1(1). WWW address http://www.epub.org.br/cm/n01/memo/remembering.htm.

[15] Buzan T, Buzan B. The mind map book: how to use radiant thinking to maximize your brain's untapped potential. Plume, 1996.

[16] Freeman E, Fertig S. Lifestreams: organizing your electronic life. AAAI Fall Symposium: AI Applications in Knowledge Navigation and Retrieval. Cambridge, MA; MIT Press, November 1995.

[17] Natrificial Software Technologies. The Brain: Information Management Software, 1998. WWW address http://www.thebrain.com.

[18] The World Wide Web Consortium. Extensible Markup Language (XML). WWW address http://www.w3.org/xml, 1997.

[19] Kobsa A. User Modeling: recent work, prospects and hazards. In: Kühme $\mathrm{T}$, Schneider-Hufschmidt $\mathrm{M}$, Malinowski U, editors. Adaptive user interfaces: principles and practice. Amsterdam: North-Holland, 1993. p. 111-28.

[20] McTear M, editor. Artificial intelligence review, special issue on user modeling, vol. 7(3). Dordrecht: Kluwer Academic Publishers, 1993.

[21] Shneiderman B. Designing the user interface. third ed. Reading, MA: Addison-Wesley and New York: Longman, 1998.

[22] Looi T, He Z. Seen by the eye but not by the mind, when attention fails. Toward a Science of Consciousness 1998 Tucson III, Arizona, April 1998.

[23] Bier E, Stone M, Fishkin K, Buxton W, Baudel T. A Taxonomy of See-Through Tools. Proceedings of CHI'94, Boston, MA, April 1994, p. 358-64.

[24] Bier E, Stone M, Pier K, Buxton W, DeRose T. Toolglass and magic lens: the see-through interface. SIGGRAPH Computer Graphics Proceedings, Annual Conference Series, Anaheim, California, August 1993, p. 73-80.

[25] Fishkin K, Stone M. Enhanced dynamic queries via movable filters. ACM Conference on Human Factors in Computing Systems CHI'95, Denver, Colorado, May 1995.

[26] Stone M, Fishkin K, Bier E. The movable filter as a user interface tool. Proceedings of CHI'94, Boston, MA, April 1994, p. 306-12.

[27] McFadden F, Hoffer J, Prescott M. Modern database management, fifth ed. Addison-Wesley, Reading, MA: 1998.

[28] Abbott K, Sarin S. Experiences with workflow management: issues for the next generation. Proceedings of the Conference on CSCW, ACM, Chapel Hill, USA, p. 113-20.

[29] Medina-Mora R, Winograd T, Flores R, Flores F. The action workflow approach to workflow management. Proceedings of the Conference on CSCW, ACM, Toronto, Canada, 1992, p. 281-88.

[30] Schäl T. Workflow management systems for process organisations. Berlin: Springer, 1996.

[31] Suchman L. Do categories have politics? The language/ action perspective reconsidered. Computer Supported Cooperative Work 1994;2(3):177-90.

[32] Winograd T. Categories, disciplines and social coordination. Computer Supported Cooperative Work 1994;2(3): 177-90. 
[33] Norman DA, Draper S, editors. User centered system design : new perspectives on human-computer interaction. Hillsdale: NJ: Lawrence Erlbaum Associates, 1986.

[34] Press L. Technology in bloom: implications for the next 50 years. Communications of the ACM 1997;40(2): $11-7$.

[35] Card S, Moran T. User Technology: From Pointing to Pondering. Proc. ACM Conference on History of Personal Workstations, 1986, p. 183-98.
[36] Nassi I, Shneiderman B. Flowchart techniques for structured programming. ACM SIGPLAN Notices, August 1973;8(8):12-26.

[37] Minas M, Shklar L. Visualizing information repositories on the world-wide web. Proceedings of the fifth International Symposium for Information Science, ISI'96, Berlin, October 1996, p. 297-309.

[38] Rassmussen J. Information processing and humanmachine interaction. Amsterdam: North-Holland, 1986. 